\title{
The shape of impacts to come: lessons and opportunities for adaptation from uneven increases in global and regional temperatures
}

\author{
Kristie L. Ebi ${ }^{1} \cdot$ Lewis H. Ziska ${ }^{2} \cdot$ Gary W. Yohe $^{3}$
}

Received: 25 April 2016 / Accepted: 22 September 2016 / Published online: 3 October 2016

(C) Springer Science+Business Media Dordrecht 2016

\begin{abstract}
Uneven patterns in the rate of climate change have profound implications for adaptation. Assuming a linear or monotonic increase in global or regional temperatures can lead to inefficient planning processes that underestimate the magnitude, pattern, and timing of the risks faced by human and natural systems, which could exaggerate future impacts and the costs of managing them. Adaptation planning needs to move beyond imposing linear thinking and analysis onto nonlinear systems. Doing so would improve research into adaptive management processes that learn from and adapt to new knowledge at a pace that reflects nonlinearity. Specifically, the pace of adaptation must consider the potential consequences of uneven increases in weather and climate variables as a means to reduce system vulnerability. Projections simulating periods of relative stability with those of rapid change would lead to more complex and more accurate expectations of future risks and associated consequences for human and natural systems. Adaptation planning based on such projections could then consider the implications of non-linear climate change on the extent of any adaptation effort, including quantified (or qualitative) risks and associated costs and benefits. Adaptation planning could be improved by projections that incorporate more nuanced understandings of how development processes could interact with climate change to alter future risks and vulnerabilities. Two examples are highlighted to illustrate the complexity and dynamic nature of non-monotonic climate-development-response scenarios: vector borne diseases and agricultural productivity.
\end{abstract}

Keywords Climate change $\cdot$ Adaptation $\cdot$ Global warming hiatus $\cdot$ Surface warming slowdown

Kristie L. Ebi

krisebi@uw.edu

1 Center for Health and the Global Environment, University of Washington, Seattle, WA 98105, USA

2 United States Department of Agriculture, Beltsville, MD 20705, USA

3 Wesleyan University, Middletown, CT 06459, USA 


\section{Introduction}

Lessons about opportunities and challenges for climate change adaptation can be drawn from observations regarding temporal variation in the rate of change of weather and climate variables, such as global mean surface temperature increases (aka the global warming "hiatus" or warming slowdown) and associated changes in the frequency and intensity of extreme precipitation events (heavy downpours and flooding or droughts associated with persistent heat extremes).

That the global mean temperature record over the past century shows considerable annual variability distributed around a persistent long term warming trend is well documented (IPCC 2013). Jones (2012) shows that this pattern of warming is less a smooth linear trend than it is a series of step changes with periods of relative stability alternating with periods during which the rates of temperature increase exceed the long-term trend. For example, the reduced rate of worldwide surface warming over the period 2001-2013, a period during which anthropogenic forcing was relatively constant, contrasts with the rapid increase in global surface temperatures since 2014 (Fyfe et al. 2016) with July $20160.8^{\circ} \mathrm{C}$ hotter than the 1951-1980 average (http:// data.giss.nasa.gov/gistemp/tabledata_v3/GLB. Ts+dSST.txt).

This sudden shift highlights the consequences of inappropriate linear analyses in assessing the exposure/risk and response capacity of natural and human systems, especially when considered in the context of similarly abrupt changes in fundamental drivers like precipitation. We are concerned that the scientific focus on determining (1) the basis for variations in the rise of global mean surface temperature with climate change and (2) whether the rate of variation per se may change (Fyfe et al. 2016; Karl et al. 2015) misses the implications of such a pattern for impacts and adaptation. These implications could be potentially profound; indeed, their significance is complementary to the underlying scientific questions.

The presumption of linearity in the climate system's response to increasing greenhouse gas concentrations is of obvious value in first order modeling of future risks; indeed, this has been the basis for nearly all adaptation research and planning focused on long-term climate change (IPCC 2014). Future risks will result from the interaction of changing hazards associated with climate change, the human and natural systems exposed to those hazards, and the vulnerability of these systems to exposure (IPCC 2012). Yet, projections of the climatic drivers of these risks do not necessarily include non-linear changes in weather and climate variables that are and will continue to be experienced by human and natural systems. Nor do they accommodate likely non-linear responses by human and natural system.

We argue here that incorporating non-linear changes in weather and climate variables (including sea level rise) in response to climate change could improve our understanding of how climate could interact with exposure and vulnerability of human systems to create risks. We focus on public health and food security, but the list of applicable sectors is much longer. We stress that, regardless of context, comparing and contrasting times of relative stability with (1) those of rapid change, (2) the contiguous abilities of adaptive research efforts to respond to new data on linear climate trends and non-linear interruptions, and (3) the capacities of communities and disciplines to plan to reduce system vulnerability, would enhance human and natural welfare (Jones 2012; O’Neill et al. 2015). Further, periods of relative stability offer opportunities for developing and testing adaptation interventions, to determine their efficacy and efficiency during periods when the urgency of adaptation is likely to be somewhat lessened. 
To that end, we draw attention to general concerns about imposing linear thinking and analysis on non-linear systems and highlight some of the advantages of moving from thinking about thresholds to thinking about a more dynamic framing of risks and responses. We argue that enhancing resilience to gradually changing average conditions underestimates exposure and therefore the potential of reducing vulnerability, especially when the patterns of impacts suddenly shift. We illustrate these issues by providing two examples - one reflects the complexity of non-linear climate change for pathogens and disease vectors; the other considers parallel implications for agriculture productivity. We end with a brief discussion of the value of incorporating complex risk profiles that consider local and development dependent contexts into adaptation planning. We do not, however, consider the value of incorporating mitigation planning into the decision framework - an important consideration, but one for another time.

\section{Imposing linear thinking and analysis on non-linear systems}

Exposure-response functions underlying impacts models are typically constructed using analytic techniques designed to identify linear relationships between a weather/climate variable (e.g. temperature and precipitation) and the response of interest. Projected increases in climate change-associated risks are often then determined by measuring the change in the response (yaxis) associated with a delta increase between the current and a future temperature (x-axis). Heat-related mortality and agricultural yields are but two examples. For example, one might report that: "If temperature were to increase by X; then Y additional deaths (+/- an error term) would result". But what would we learn if the results were framed as "If temperature were to increase by $\mathrm{x} \%$, then $\mathrm{Y}$ would increase by $\mathrm{y} \%$ "? In the first conclusion, the answer is a constant slope linking $\mathrm{X}$ and $\mathrm{Y}$. In the second, the answer is a slope linking a percentage change in $\mathrm{X}$ with a percentage change in $\mathrm{Y}$ - an elasticity estimate. The elasticity estimate could be zero, in which case all of this is only of academic interest. The elasticity estimate could, however, be statistically greater than (less than) zero, in which case the change in $Y$ for every change in X could accelerate (or, alternatively, decelerate).

While a linear function might be a good first step in estimating risk relative to impact and the subsequent value of adaption, the simple arithmetic of the previous paragraph suggests the linear approach ignores the often-complex relationships between exposure and response (likelihood and net consequence). Specifically, it may overlook the tails of the distribution where relationships may be highly non-linear (e.g. extremes). This does not, however, mean that the tails are the only challenges. Models that incorporate non-linear climate events (e.g. temperature) and subsequent system responses may provide more realistic estimates of climate change risks over temporal scales even within the middle of the distribution of possible futures.

Adaption should also be considered in terms of the temporal scales of their efficacy. It would be helpful, for example, to explore vulnerabilities driven by temperature increases without adaptation against scenarios within which systems have adapted not only to the anticipated linear trend, but also to the acknowledged non-linear acceleration of those trends. Only half of the temperature increase to which we have committed ourselves is reflected in temperature change; the other $50 \%$ amplifies observed temperature increases at an increasing rate.

The temporal implications of this recent scientific conclusion are not always intuitive. While it seems as if this delayed climate system response is a long-term issue, the opposite is possible. For example, adaptation and development, informed by long-term trends alone, could 
significantly reduce malaria incidence in the long run, but the short-term (5-10 years) impacts of temperature and precipitation events on the distribution of the Anopheles mosquito that carries malaria (without additional adaptation) could mean large numbers of preventable childhood deaths (Caminade et al. 2014; Smith et al. 2014).

\section{The shapes of response when thresholds are the sources of non-linearity}

The climate change literature is replete with references to the importance of understanding thresholds where changing temperature, precipitation, sea level rise, ocean acidification, inter alia, could result in system transition (see IPCC 2014). These discussions take ecosystem collapse or changes in geophysical states as their model, i.e. when a changed parameter or condition results in a sudden (and often unexpected) shift in biological or physical transformation. Examples of such "tipping points" include extinctions, including species extinctions that could open ecological niches for other species to expand, fishery collapse, pathogen or invasive shifts with sudden loss of diversity, or rapid sea level rise (e.g. sudden melting of the West Antarctic ice sheet). While these sorts of dramatic responses can occur, a more subtle or complex response is also possible. For example, the near collapse of species or ecosystems can lead to some level of recovery by changes in their geographic range characterized by gradual excursions instead of sudden appearances or disappearances (Parmesan and Yohe 2003; Root et al. 2003).

The temporal and transient nature of climate change impacts should also be considered in the context of the many social, economic, and environmental drivers in human and natural systems. Consequently, whether a particular climatic change will cause a threshold shift will also depend on other factors, including the extent to which projected climate change is effectively incorporated into existing adaptation and mitigation strategies.

Implementation of adaptation and mitigation strategies should also consider the heterogeneity of future risks on different populations and social groups, particularly the effectiveness of adaptation for vulnerable groups with low political or economic power to influence adaptation decision-making. Hence, preparations for, and responses to, climate change-related risks will also be non-linear. The extent to which future societies invest in scientific research and development will likely be as uneven as historic investments. Therefore, the adaptation knowledge, methods, and tools available to future societies are fundamentally uncertain, although insights into possible future shapes of scientific insight and discovery could be developed from the narratives of the shared socioeconomic pathways (O'Neill et al. 2015).

Overall, projecting realistic climate change risks and impacts requires moving from simplistic models where climate drives linear outcomes to more nuanced models of the spatial and temporal interplay of climate drivers, including the hazards associated with a changing climate, who or what is exposed to those changes, their sensitivities to exposure, and the capacity of individuals and societies to implement adaptation and mitigation policies (IPCC 2012; NRC 2013). Two examples, chosen because of expertise of the authors and because quality data are available to support multiple analyses, follow.

\subsection{Pathogens and disease}

Climate change could facilitate changes in the incidence, geographic range, and seasonality of a large number of vectorborne diseases (Smith et al. 2014). Using malaria as an example, 
climate is one driver that affects the altitudinal range of the vector (various species of Anopheles) that carries the malaria pathogen. Other anthropogenic factors also affect this range, including land use change, drug resistance, human migration, status of health systems, inter alia. Modeling based on laboratory understanding of the temperature and precipitation requirements of Anopheles and plasmodium (the malaria pathogen) for reproduction and disease transmission shows that small changes in temperature can lead to a very large increase in the number of vectors, a known risk factor for the number of malaria cases (Pascual et al. 2006).

If detailed information on vector distribution were available, it would then be possible to use these relationships to project how non-linear increases in temperatures with climate change could increase the numbers of mosquitoes over time in highland areas (Parham and Michael 2010). Because populations above certain altitudes do not have immunity to malaria (which develops following repeated exposure to the pathogen, such as occurs in endemic regions), introduction of the malarial parasite above a threshold altitude would be expected to result (depending on the extent of anticipatory adaptation) in epidemics with higher mortality (up to about $10 \%$, spread across all age ranges, instead of less than $3 \%$, mostly in children, in endemic regions). Further, if there were inadequate surveillance, then initial epidemics would have a larger impact and take longer to bring under control. That is, an increase in temperature above a certain elevation and the extent of malarial mortality would not follow a monotonic and linear response function.

National and international organizations and institutions would be expected to step in when mortality rates began to rise in highland regions, to implement malaria control programs known to be successful in reducing the number of cases, such as through providing insecticide treated bed nets, residual insecticide spraying, and integrated vector management. Ideally, these organizations and institutions would have been monitoring the risks of malaria changing its geographic range due to climate change and would have implemented proactive surveillance programs and early warning systems so that high mortality rates could have been avoided in the first place. Gradually, the risk of major epidemics would be expected to decrease even as the climate continues to change because of these control programs. Further, as epidemics occur more and more regularly, malaria becomes endemic, which means the disease is less deadly to adults and the focus shifts to controlling the exposures of children and pregnant women.

In addition, there can be a temporal shift in the shape of the climate-malaria response curve that may reflect reduced incidence. Concurrent with rising temperature and altitudinal changes, some endemic regions could become too hot or too dry for Anopheles, resulting in a sudden decline in the burden of malaria.

Adding these additional non-monotonic considerations into impacts models would be invaluable to decision-makers to inform how incidence could change quickly and to provide a more nuanced understanding of possible risks and appropriate adaptive responses, including an estimate of the human and financial resources that would be needed. At present, inclusion of these temporal changes in modeling the evolution of malaria in the context of climate change are limited because doing so is challenging, with no agreed approaches.

Development choices also matter (Ebi 2014). Overall, development pathways that are directed to improve public health infrastructure (e.g. improved sanitation, safe water, education) are also unlikely to be characterized by slow and steady (e.g. linear) change over time. The rate of progress in improving conditions that contribute to the burden of malaria will provide the baseline vulnerability with which climate hazards will interact. 
Malaria of course, is not the only vectorborne disease whose geographic range and intensity of transmission will be affected by climate change. The bio-geographical range and incidence of other diseases such as the Zika virus can also transition quickly with rapid temperature and/ or precipitation changes. As with malaria, the public health responses to such diseases are influenced by infrastructure patterns, technological developments, human and animal behaviors, capabilities of health systems, governance, political will, inter alia. These responses in turn, will mirror the extent of disease transmission and are also unlikely to be monotonic.

However, it is also important to emphasize that pathogens and associated vectors may not move concurrently, and that differential responses of pathogen/vector to weather or climate may affect the timing of transmission emergence. For example, in Sweden, the ticks that can carry the tick-borne encephalitis (TBE) virus (Ixodes ricinus and I. persulcatus) are increasing their range, due, in part, to milder winters and a longer growing season that supports the survival and proliferation of the tick and its host (generally roe deer) (Jaenson et al. 2012). There needs to be sufficient numbers of infected ticks over time for the virus to become established. Therefore, there will be a temporal lag between establishment of infected ticks and occurrence of disease. From the perspective of temperature increases as a driver of the process, periods of rapid increase in temperature can offer opportunities for ticks to swiftly move to new environments, while the subsequent more stable period may allow for tick density to increase, the virus to become established, and transmission to begin. Better incorporation of the temporal dynamic into adaptation planning can move surveillance programs from focusing on detecting human cases to improving monitoring of the bio-climatic edges of current tick and deer distribution.

\subsection{Agricultural productivity}

Globally, agriculture engages in climate risk assessment just prior to planting, i.e. management decisions are made that attempt to maximize production and minimize environmental risk for a given set of weather conditions. For example, if the fields are wet, planting dates can be delayed; similarly, changes in fertilizer, tillage, crop variety etc. can compensate for a wetter than normal environment. In general, such practices are effective in regard to adaptation of regional weather events because agricultural risk management practices are, by their nature, characterized by crop specific environments.

There are, however, limits to the resiliency of such decisions in regard to long-term temporal shifts in environmental extremes. That is, if a weather event (short-term) becomes a climate event (long-term), traditional once-a-season planning risk assessments may be inadequate. Simple climate impact models do not always capture the non-linearity of such events. For example, rapid (days, weeks) exposure to high temperature does not result in linear production losses; rather, for many essential crop species including rice, wheat, and corn, yield collapse occurs if temperatures exceed reproductive thermal thresholds even for one or two days (Backlund et al. 2008; Schlenker and Roberts 2009). Similarly, rapid occurrence of drought (e.g. "flash" droughts) can result in similar yield collapse, particularly if the drought occurs during flowering (Hatfield et al. 2011).

Climate imposes significant risks of sudden, persistent shifts in the physical environment (e.g. drought, temperature extremes) that highlight the vulnerability of agricultural production and why managing the temporal uncertainty of climate change will be important for achieving food security (Ziska 2011; Burke et al. 2015). In that regard, understanding and identifying vulnerability is unlikely to be met through the use of traditional, single season, monotonic 
response models. For example, it cannot be assumed that a cumulative precipitation of $\mathrm{x}$ results in a yield of $y$, if, temporally, seasonal precipitation now occurs over a short period. Such enhanced in-season climactic uncertainty illustrates the need for model complexity in assessing risk and implementing new adaptation practices that maintain production resilience even with dynamic temporal changes in climate (Lobell et al. 2014).

At the farm level, adaptation should, if feasible, promote spatial and temporal diversity in genetics and management as a means to reduce the consequences of climatic shifts (Ziska 2011). This could include increased availability of climate resistant varieties; e.g. drought tolerant lines or consideration of non-traditional crops associated with changing agro-climatic zones. Management decisions that improve resource capture, such as the use of no-till, the planting of cover crops or augmented crop rotation, implementation of planned-deficit irrigation, may also increase production resiliency to extreme climatic events. Strengthening or improving infrastructure capacity, such as installation of irrigation systems, tile drains or improved rainfall capture, could also help to increase production resilience (Howden et al. 2007; Lobell et al. 2008). At the regional or national level, development or improvement of climate forecasting, as well as improvement in the detection and tracking of existing and emerging pest threats in agriculture and forestry (e.g. Pine bark beetle), can help in maintaining the viability of production systems.

At present, however, considerations of genetic and spatial crop diversity, enhanced pest threats, improved infrastructure, greater resource efficiency, and effective forecasting are, for the most part, lacking as means to reduce the production risks associated with sudden temporal shifts in climate. Given the time period associated with implementation of such practices (e.g. a new crop variety may take 10-15 years before available) and the acknowledgment that monotonic change is unlikely (Cowtan and Way 2014; England et al. 2014), there is an insistent need to investigate, develop, and incorporate non-linear complexity in current agricultural adaptation efforts.

\section{Considerations and conclusions}

We argue, here, that adaptation planning and implementation for climate change based on assumptions of monotonic and linear increments in environmental parameters such as temperature will leave future natural and human systems ill-prepared to cope with and manage the long-term temporal risks. Current modeling efforts to assess impacts and adaptation generally assume gradual shifts to higher (or lower) values of mean temperature or precipitation, with gradual changes in impacts, at least until thresholds (climatic, social, or both) are crossed and nonlinearities emerge. However, non-linear shifts in the rate of temperature increase, for example, can amplify manifestations of associated damage and highlight vulnerability (IPCC 2012).

We provided two examples, one related to vectorborne diseases and the other related to agricultural productivity in the context of rapid climatic shifts. These examples are not meant to be exclusive and exhaustive. They are, instead, included to illustrate more dynamic temporal change in the physical climate as well as the consequences for the adaptive response in human systems.

If climatic impacts and subsequent costs to agriculture, public health, and other sectors are to be avoided, then the pace of effective adaptation measures must consider the non-linearity of climatic change. The specific means by which such non-linearity could be included will 
depend on the system being considered (e.g. human health). Iterative risk management is flexible enough to incorporate non-linear risks into planning processes that consider periods when there are opportunities to consolidate gains yet prepare for periods of more intense impacts. Taking non-linear change as the point of departure for adaptation pathways offers opportunities and challenges as the perceived urgency of adaptation waxes and wanes. Periods of relative stability offer opportunities for consolidating earlier gains from adaptation efforts and for innovating new adaptation interventions, with time for testing which options would likely be robust against coming periods of rapid change. The impacts of periods of rapid change would be lessened by proactive adaptation designed to manage these transitions.

Although pauses in long-term climate change may create opportunities for some systems to adjust to changes that have already occurred, complacency in planning and/or implementation of adaptation could also arise. The assumption that climate change is linear or monotonic can lead planners to adjust the subjective probabilities they attach to climate projections. Such adjustments could limit adaptation research, potentially at an enormous cost. In turn, this may amplify initial damages, especially in cases where the perceived level of risk is generated by stochastic variability with rising temperatures (e.g. drought and agriculture). Adaptation planners that effectively communicate the non-linear nature of climate risks to the communities they serve are more likely to gain support for earlier, lower cost interventions.

Developing impact models that move beyond linear assumptions of increases in weather and climate variables to explicitly consider non-linear rates of change will be difficult. Incorporating a range of temporally dynamic pathways will provide more nuanced and useful projections of climatic risks within the context of other drivers of impacts, and, therefore, the opportunity to develop more effective and efficient solutions to protect human and natural systems as climate and development proceed. At the same time, explicitly incorporating the dynamic nature of adaptation responses, while complex, could provide more realistic assessments of the pattern of challenges and opportunities ahead.

\section{References}

Backlund P et al (2008) The effects of climate change on agriculture, land resources, water resources, and biodiversity in the United States. Final Report-Synthesis and Assessment Product 4.3. A Report by the U.S. Climate Change Science Program and the Subcommittee on Global Change Research. Washington, DC: U.S. Department of Agriculture, $250 \mathrm{pp}$.

Burke $\mathrm{M}$ et al (2015) Incorporating climate uncertainty into estimates of climate change impacts. Rev Econ Stat 97(2):461-471

Caminade C et al (2014) Impact of climate change on global malaria distribution. Proc Natl Acad Sci 111:32863291. doi:10.1073/pnas.1302089111

Cowtan K, Way RG (2014) Coverage bias in the HadCRUT4 temperature series and its impact on recent temperature trends. Q J R Meteorol Soc 140:1935-1944

Ebi KL (2014) Health in the new scenarios for climate change research. Int J Environ Res Public Health 10. doi: 10.3390/ijerph100x000x

England MH et al (2014) Recent intensification of wind-driven circulation in the Pacific and the ongoing warming hiatus. Nat Clim Chang 4:222-227

Fyfe JC et al (2016) Making sense of the early 2000s warming slowdown. Nat Clim Chang 6:224-228

Hatfield JL et al (2011) Climate impacts on agriculture: implications for crop production. Agron J 103:351-370

Howden SM et al (2007) Adapting agriculture to climate change. Proc Natl Acad Sci 104:19691-19696

IPCC (2012) Managing the risks of extreme events and disasters to advance climate change adaptation. A special report of working groups I and II of the intergovernmental panel on climate change. In: Field CB et al (eds) Cambridge University Press, Cambridge and New York, 582 pp 
IPCC (2013) Summary for policymakers. In: Climate Change 2013: The physical science basis. Contribution of working group I to the fifth assessment report of the intergovernmental panel on climate change. In: Stocker, $\mathrm{TF}$ et al (eds) Cambridge University Press, Cambridge and New York, $28 \mathrm{pp}$

IPCC (2014) Summary for policymakers. In: Climate Change 2014: Impacts, adaptation, and vulnerability. Part A: Global and sectoral aspects. Contribution of working Group II to the fifth assessment report of the intergovernmental panel on climate change. In: Field CB et al (eds) Cambridge University Press, Cambridge and New York, $32 \mathrm{pp}$

Jaenson TGT, Jaenson DGE, Eisen L, Petersson E, Lindgren E (2012) Change in the geographic distribution and abundance of the tick Ixodes ricinus during the past 30 years in Sweden. Parasit Vectors 5:8

Jones RN (2012) Detecting and attributing nonlinear anthropogenic regional warming in southeastern Australia. J Geophys Res 117:D04105

Karl TR et al (2015) Possible artifacts of data biases in the recent global surface warming hiatus. Science 348: 1469-1472

Lobell DB et al (2008) Prioritizing climate change adaptation needs for food security in 2030. Science 319:607610

Lobell DB et al (2014) Greater sensitivity to drought accompanies maize yield increase in the US Midwest. Science 344(6183):516-519

NRC (2013) Steinbruner JD, Stern PC, Husbands JL (eds) Climate and social stress: implications for security analysis. Committee on assessing the impacts of climate change on social and political stresses. Board on Environmental Change and Society, Division of Behavioral and Social Sciences and Education, The National Academies Press, Washington, DC, $280 \mathrm{pp}$

O’Neill BC et al (2015) The roads ahead: narratives for shared socioeconomic pathways describing world futures in the twenty-first century. Glob Environ Chang doi:10.1016/j.gloenvcha.2015.01.004

Parham PE, Michael E (2010) Modeling the effects of weather and climate change on malaria transmission. Environ Health Perspect 118:620-626

Parmesan C, Yohe G (2003) A globally coherent fingerprint of climate change imacts across natural systems. Nature 421:37-42

Pascual M et al (2006) Malaria resurgence in the east African highlands: temperature trends revisited. Proc Natl Acad Sci 15:5829-5834

Root TL et al (2003) Fingerprints of global warming on wild animals and plants. Nature 421:57-60

Schlenker W, Roberts MJ (2009) Nonlinear temperature effects include severe damages to U.S. corn yields under climate change. Proc Natl Acad Sci 106:15594-15598

Smith KR et al (2014) Human health: impacts, adaptation, and co-benefits. In: Climate Change 2014: Impacts, adaptation, and vulnerability. Part A: Global and sectoral aspects. Contribution of working group II to the fifth assessment report of the intergovernmental panel on climate change. In: Field CB et al (eds) Cambridge University Press, Cambridge and New York, pp 709-754

Ziska LH (2011) Climate change, carbon dioxide and global crop production: food security and uncertainty. In: Handbook on Climate Change and Agriculture, Dinar A, Mendelsohn R (eds), Edward Elgar Publishing, Cheltenham, pages 9-31 